\title{
A randomised trial of voriconazole and prednisolone monotherapy in acute-stage allergic bronchopulmonary aspergillosis complicating asthma
}

\author{
To the Editor:
}

Glucocorticoids are the most widely used agents in the treatment of allergic bronchopulmonary aspergillosis (ABPA), a disorder characterised by immunologic reactions mounted against Aspergillus fumigatus colonising the airways of patients with asthma and cystic fibrosis [1, 2]. Unfortunately, the use of glucocorticoids is associated with several adverse reactions [3]. A novel treatment strategy in ABPA would be the use of antifungal triazoles as monotherapy. Recently, we have shown that itraconazole was effective as monotherapy in acute-stage ABPA [4]. Whether voriconazole monotherapy is also efficacious in acute-stage ABPA remains unknown.

We conducted a single-centre, unblinded, randomised controlled trial between January 2014 and July 2015 in the Chest Clinic of the Postgraduate Institute of Medical Education and Research (PGIMER). The Institute Ethics Committee approved the study protocol and written consent was obtained from all subjects. We included consecutive subjects of ABPA if they met all of the following criteria: 1) asthma; 2) immediate cutaneous hyperreactivity to Aspergillus antigen; 3) elevated total IgE $>1000 \mathrm{IU} \cdot \mathrm{mL}^{-1}$; 4) A. fumigatus-specific IgE $>0.35 \mathrm{kUA} \cdot \mathrm{L}^{-1}$ and two of the following criteria: 1) presence of serum precipitins against A. fumigatus; 2) fixed or transient radiographic pulmonary opacities; 3) peripheral blood eosinophil count $>1000$ cells $\mu \mathrm{L}^{-1}$; 4) bronchiectasis on computed tomography (CT) chest scan [5]. We excluded subjects for the following criteria: 1) failure to provide consent; 2) intake of systemic glucocorticoids or azoles for $>3$ weeks in the preceding 6 months; 3) omalizumab therapy; 4) immunosuppressive therapy and immunosuppressive states; 5) enrolment in another trial of ABPA; and 6) pregnancy.

Subjects were randomised sequentially 1:1 to either the voriconazole group or the glucocorticoid group using a computer-generated randomisation sequence (assignments were placed in opaque sealed envelopes) (figure 1). Patients in the glucocorticoid arm received oral prednisolone (Omnacortil tablets, Macleod's Pharmaceuticals, India) at $0.5 \mathrm{mg} \cdot \mathrm{kg}^{-1} \cdot \mathrm{day}^{-1}$ for 4 weeks; $0.25 \mathrm{mg} \cdot \mathrm{kg}^{-1} \cdot \mathrm{day}^{-1}$ for 4 weeks; $0.125 \mathrm{mg} \cdot \mathrm{kg}^{-1} \cdot \mathrm{day}^{-1}$ for 4 weeks; then tapered by $5 \mathrm{mg} \cdot\left(2\right.$ weeks) ${ }^{-1}$ and discontinued (total duration: 4 months). In the voriconazole arm, subjects received oral voriconazole (Voritek capsules, Cipla Pharmaceuticals, India) at $200 \mathrm{mg}$ twice daily, $1 \mathrm{~h}$ before or after a meal, for 4 months. Adherence to therapy was ensured by instructing patients to bring the empty pill covers. We also measured voriconazole levels at 6 weeks and 3 months. For the control of asthma, treatment with inhaled corticosteroids (ICS), long-acting $\beta 2$-agonists (formoterol) and montelukast was allowed in both groups. We recorded demographic, clinical, immunological, spirometric and radiological details at the baseline visit. The subjects were followed with clinical examination, chest radiograph and serum total IgE every 6 weeks for 6 months. At the first follow-up visit, we also performed spirometry and assessed health status using the St. George's Respiratory Questionnaire (SGRQ) score. Subsequently, the patients were followed-up every 6 months, or earlier if there was worsening in symptoms. We clinically evaluated all the subjects for

@ERSpublications

Voriconazole monotherapy is as effective, and safer, than glucocorticoids for the treatment of ABPA http://ow.ly/jCix3012Lb9

Cite this article as: Agarwal R, Dhooria S, Sehgal IS, et al. A randomised trial of voriconazole and prednisolone monotherapy in acute-stage allergic bronchopulmonary aspergillosis complicating asthma. Eur Respir J 2018; 52: 1801159 [https://doi.org/10.1183/13993003.01159-2018]. 
a)
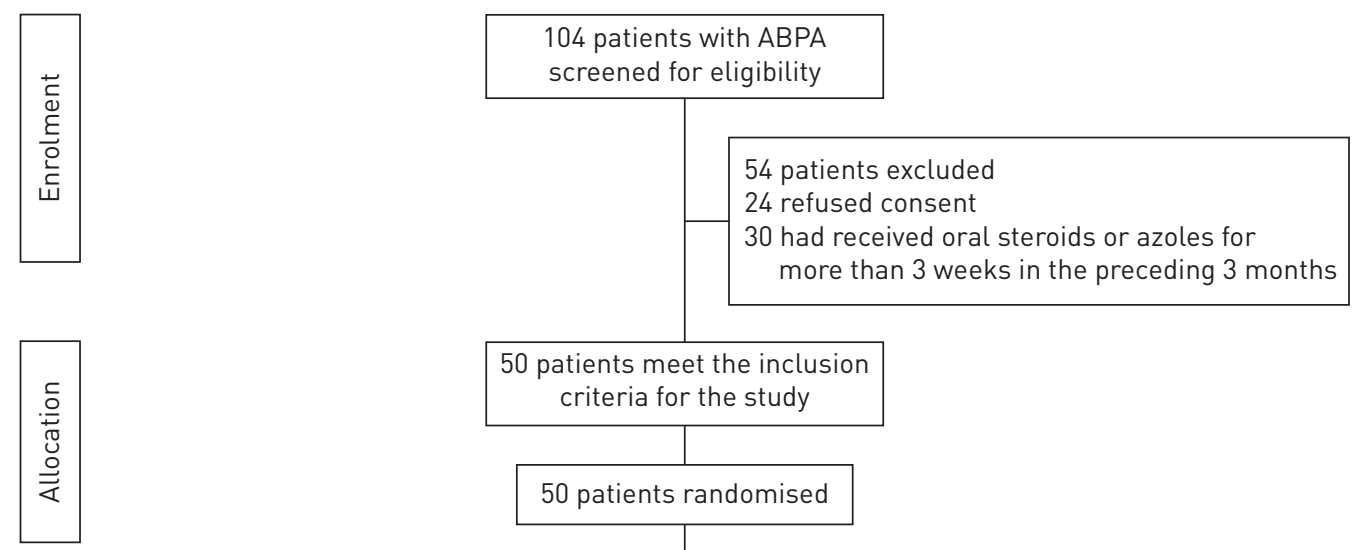

50 patients meet the inclusion criteria for the study

50 patients randomised

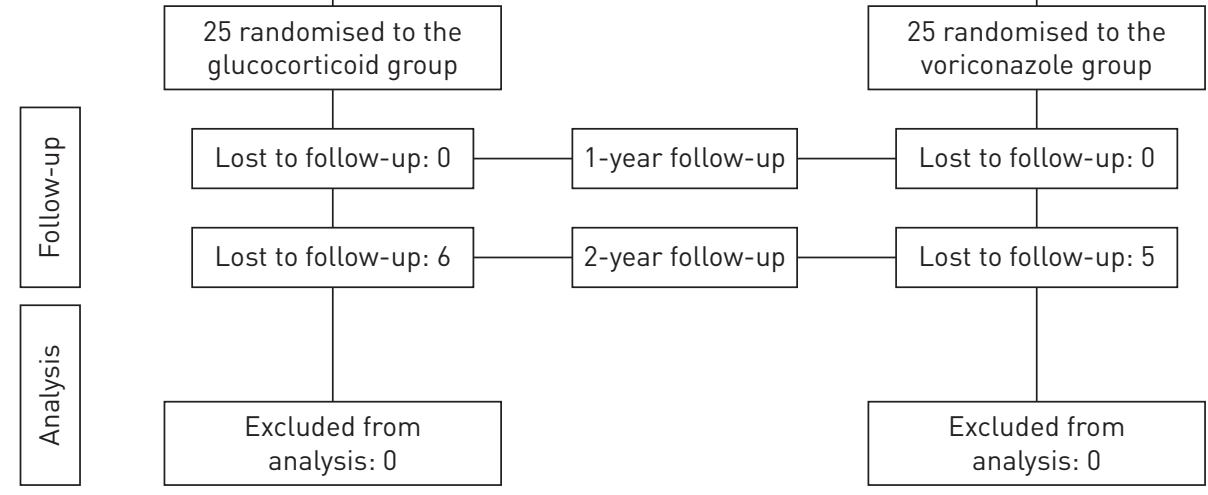

b)

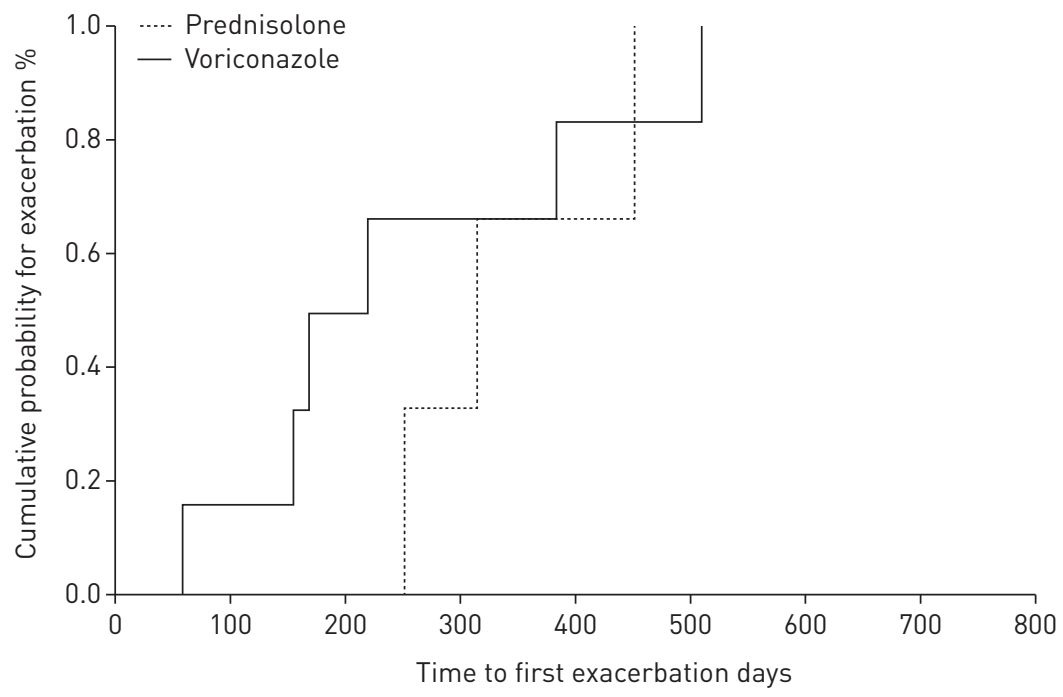

Number at risk

Prednisolone

Voriconazole

Number on follow-up

Prednisolone

Voriconazole

$\begin{array}{lllllllll}3 & 3 & 3 & 2 & 1 & 0 & 0 & 0 & 0 \\ 6 & 5 & 3 & 2 & 1 & 1 & 0 & 0 & 0 \\ 25 & 25 & 25 & 25 & 25 & 20 & 19 & 19 & 19 \\ 25 & 25 & 25 & 25 & 25 & 22 & 21 & 20 & 20\end{array}$

FIGURE 1 a) CONSORT diagram demonstrating the flow of participants in the study; b) time to first exacerbation in subjects receiving voriconazole (solid line) versus prednisolone (dotted line) in subjects with allergic bronchopulmonary aspergillosis (ABPA). The time to first exacerbation was similar in the two groups. The number at risk in each group denotes the numbers of patients still at risk of experiencing the first exacerbation. 
adverse reactions and also monitored fasting plasma glucose, blood pressure and liver function every 6 weeks.

The treatment effects were classified as: 1) composite response: improvement in cough and dyspnoea ( $>75 \%$ of baseline) accompanied by at least $50 \%$ clearance of chest radiographic opacities (if present prior to treatment initiation) along with decline in serum total $\operatorname{IgE}$ by $\geqslant 25 \%$, after 6 weeks of treatment $[4,6]$; 2) ABPA exacerbation: clinical and/or radiological worsening along with doubling of the serum total $\operatorname{IgE}$ over the previous baseline value; and 3) asthma exacerbation: clinical worsening in cough and dyspnoea with no radiological worsening or doubling of serum total IgE. The first ABPA exacerbation in both arms was treated with oral prednisolone at a dose of $0.5 \mathrm{mg} \cdot \mathrm{kg}^{-1} \cdot \mathrm{day}^{-1}$ for 4 weeks; $0.25 \mathrm{mg} \cdot \mathrm{kg}^{-1} \cdot \mathrm{day}^{-1}$ for 4 weeks; $0.125 \mathrm{mg} \cdot \mathrm{kg}^{-1} \cdot \mathrm{day}^{-1}$ for 4 weeks; then decreased by $5 \mathrm{mg} \cdot\left(2\right.$ weeks ${ }^{-1}$ (total: 4 months). All subsequent exacerbations were treated with prednisolone at the dose above along with itraconazole at $400 \mathrm{mg} \cdot \mathrm{day}^{-1}$ for 6 months. Asthma exacerbations were treated with oral prednisolone at $0.5 \mathrm{mg} \cdot \mathrm{kg}^{-1} \cdot \mathrm{day}^{-1}$ for 5-7 days [7]. The primary outcomes were: 1) composite response rates in the two groups after initiation of treatment (6 weeks and 3 months); and 2) ABPA exacerbation rates in the two groups after completion of treatment (12 and 24 months). The secondary outcome was treatment-related adverse effects in the two groups.

Fifty subjects (figure 1) were randomised to receive either prednisolone $(n=25)$ or voriconazole $(n=25)$. Baseline characteristics, including the immunological parameters, were similar in the two groups. The mean \pm SD age of the study subjects $(72 \%$ men) was $37.4 \pm 14.1$ years. The mean duration of asthma at the time of diagnosis was 8.7 years. The severity of airflow obstruction was similar in the two groups (forced expiratory volume in $1 \mathrm{~s}\left(\mathrm{FEV}_{1}\right)$ was less than $80 \%$ in $58 \%$ of the subjects). All the subjects had

TABLE 1 Outcomes of study subjects treated with prednisolone or voriconazole

Prednisolone group

25

Subjects

\section{Primary outcomes}

Subjects with composite response

After 6 weeks of treatment

After 3 months of treatment

Subjects experiencing exacerbation

After 12 months of treatment

After 24 months of treatment

\section{Other outcomes}

Decline in IgE after 6 weeks of treatment \%

Time to first exacerbation days

Difference in FEV 1 after 6 weeks of treatment $\mathrm{mL}$

Difference in FVC after 6 weeks of treatment $\mathrm{mL}$

Change in score after 6 weeks of treatment

ABPA exacerbations

Asthma exacerbations

\section{Adverse reactions}

Cushingoid habitus

Hypertension

Hyperglycaemia

Hypertrichosis

Acne

Striae

Weight gain at 6 weeks $\%$

Weight gain $>5 \%$

Mood changes

Fatigue

Visual disturbance

Skin rash

Liver function test abnormalities

Nausea

Discontinuation of study drug

Any adverse effect
47.9 (37.3 to 58.4 )

339 (85 to 593 )

271 (191 to 350 )

312 (234 to 389 )

$-25.1(-17.9$ to -32.3$)$

0.24 (0.02 to 0.46 )

$0.36(0.13$ to 0.59$)$

$11(44.0)$
0
0
$2(8.0)$
$2(8.0)$
$1(4.0)$
$9(3.7$ to 10.3$)$
$13(52)$

0

0

0

0

0

0

0

29

Voriconazole group

Estimate difference $(95 \% \mathrm{CI})$

p-value

25

$\begin{array}{ll}25(100) & 24(96.0) \\ 25(100) & 24(96.0) \\ 2(8.0) & 3(12.0) \\ 3(12.0) & 5(20.0)\end{array}$

$0.04(-0.10$ to 0.20$)$

0.31

0.31

0.64

$0.04(-0.23$ to 0.15$)$

$0.08(-0.29$ to 0.13$)$

0.44

45.4 (36.4 to 54.4 )

248 (73 to 424$)$

370 (205 to 536 )

395 (262 to 528$)$

$-22.7(-14.2$ to -31.3$)$

$0.52(0.23$ to 0.81$)$

$0.48(0.27$ to 0.69$)$

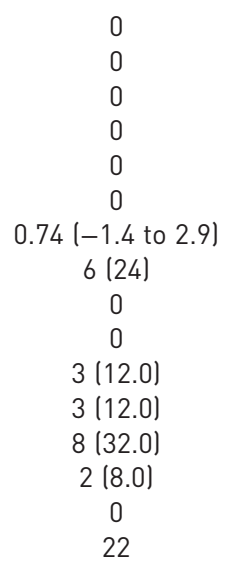

$2.48(-11.04$ to 16.00$) \quad 0.66$

$91(12$ to 170$) \quad 0.30$

$-99(-269$ to 71$) \quad 0.69$

$-83(-229$ to 63$) \quad 0.67$

$2.4(-8.3$ to 13.1$) \quad 0.99$

$-0.28(-0.63$ to 0.07$) \quad 0.12$

$-0.12(-0.43$ to 0.19$) \quad 0.32$

$0.44(0.22$ to 0.63$) \quad 0.0001$

$0.08(-0.07$ to 0.25$) \quad 0.49$

$0.08(-0.07$ to 0.25$) \quad 0.49$

$0.04(-0.10$ to 0.20$) \quad 0.99$

6.23 (2.14 to 10.05 ) $\quad 0.002$

$0.28(0.01$ to 0.50$) \quad 0.04$

$-0.12(-0.30$ to 0.04$) \quad 0.24$

$-0.12(-0.30$ to 0.04$) \quad 0.24$

$-0.32(-0.52$ to -0.12$) \quad 0.004$

$-0.08(-0.25$ to 0.07$) \quad 0.49$

All values are presented as $\mathrm{n}, \mathrm{n}(\%)$ or mean $(95 \% \mathrm{CI})$, unless otherwise stated. FEV1: forced expiratory volume in 1 s; FVC: forced vital capacity; ABPA: allergic bronchopulmonary aspergillosis. 
bronchiectasis on high-resolution computed tomography (HRCT) chest scan, while 29\% also had high-attenuation mucus. The baseline immunological parameters including serum IgE (total and A. fumigatus-specific) and eosinophil counts were similar in the two groups. All the subjects were receiving ICS (mean \pm SD dosage: $397 \pm 187 \mathrm{mg} \cdot \mathrm{day}^{-1}$ ) and long-acting $\beta_{2}$-agonists (mean \pm SD dosage: $14 \pm 6.3$ $\mathrm{mg} \cdot \mathrm{day}^{-1}$ ) while $44 \%$ were receiving montelukast. The participants were followed-up for a mean \pm SD period of $77 \pm 32$ months; however, all analyses were performed until 2 years of follow-up. The proportion of subjects demonstrating a composite response after 6 weeks and 3 months was similar in the two groups (prednisolone $(\mathrm{n}=25(100 \%))$ versus voriconazole $(\mathrm{n}=24(96 \%)) ; \mathrm{p}=0.31)$ (table 1). The trough levels of voriconazole were $>1 \mathrm{mg} \cdot \mathrm{L}^{-1},>0.5 \mathrm{mg} \cdot \mathrm{L}^{-1}$ and $<0.5 \mathrm{mg} \cdot \mathrm{L}^{-1}$ in 19 , three and three subjects, respectively. In the only subject who failed initial treatment with voriconazole, the drug levels were in the therapeutic range $\left(>1 \mathrm{mg} \cdot \mathrm{L}^{-1}\right)$. The numbers of subjects with exacerbations after 1 year and 2 years were similar in the two groups (table 1). The incidence of adverse events in the two groups is shown in table 1. The occurrence of cushingoid habitus and weight gain were significantly higher in the glucocorticoid group. Transient derangements in liver function occurred in eight subjects (32\%) in the voriconazole group. Three subjects each receiving voriconazole developed transient visual disturbance and photosensitive skin rash (which disappeared after avoidance of sunlight exposure). The percentage decline in IgE and the change in lung function were similar in the two groups after 6 weeks of treatment (table 1). There was a considerable decline in SGRQ score at 6 weeks (mean: 23.9), which was not different in the two groups (table 1). The time to first exacerbation (prednisolone (mean: 339 days) versus voriconazole (mean: 248 days)) was similar in the two groups (table 1). The total number of asthma and ABPA exacerbations was also similar in the two groups at 2-year follow-up.

Only a few randomised trials have evaluated triazoles in patients with ABPA. Two small placebo-controlled trials have previously demonstrated the usefulness of itraconazole $\left(400 \mathrm{mg}^{-\mathrm{day}^{-1}}\right.$ for 4 months) in glucocorticoid-dependent ABPA [8,9]. Recently, we have published the results of itraconazole monotherapy in acute-stage ABPA complicating asthma [4]. In that study, 131 patients with ABPA were randomised to receive either itraconazole $\left(400 \mathrm{mg}^{\left.-\mathrm{day}^{-1}\right)}\right.$ or prednisolone (at the same dose as used in the current study) for 4 months [4]. While prednisolone was superior to itraconazole in inducing a composite response, itraconazole was also effective in a considerable number of cases (88\%). The exacerbation rate at 2 years was also similar in the two groups [4]. The efficacy of voriconazole monotherapy in the current study is $96 \%$, which is higher than the efficacy of itraconazole [4]. The better outcomes with voriconazole (versus itraconazole) could be due to its better intrinsic activity against A. fumigatus, lesser incidence of resistance, or better bioavailability [10].

Finally, our study has a few limitations. This was a single-centre, unblinded study with a small sample size (156 subjects were required for the current study to have a $90 \%$ power of detecting significance at the $5 \%$ level). Thus, our conclusions are hypothesis-generating. For instance, there was a trend towards lesser time to first exacerbation and more ABPA and asthma exacerbations in the voriconazole arm, which might have proved statistically significant had we included a larger study population. Finally, we used a fixed dose of voriconazole and did not adjust the dose based on levels.

In conclusion, voriconazole appears to be as effective as glucocorticoids in acute-stage ABPA. Larger trials are required to confirm our study results.

Ritesh Agarwal $^{1}$, Sahajal Dhooria ${ }^{1}$, Inderpaul S. Sehgal ${ }^{1}$, Ashutosh N. Aggarwal ${ }^{1}$, Mandeep Garg ${ }^{2}$, Biman Saikia ${ }^{3}$ and Arunaloke Chakrabarti ${ }^{4}$

${ }^{1}$ Dept of Pulmonary Medicine, Postgraduate Institute of Medical Education and Research (PGIMER), Chandigarh, India. ${ }^{2}$ Radiodiagnosis and Imaging, Postgraduate Institute of Medical Education and Research (PGIMER), Chandigarh, India. ${ }^{3}$ Dept of Immunopathology, Postgraduate Institute of Medical Education and Research (PGIMER), Chandigarh, India. ${ }^{4}$ Dept of Medical Microbiology, Postgraduate Institute of Medical Education and Research (PGIMER), Chandigarh, India.

Correspondence: Ritesh Agarwal, Dept of Pulmonary Medicine, Postgraduate Institute of Medical Education and Research (PGIMER), Chandigarh, India 160012. E-mail: agarwal.ritesh@outlook.in

Received: April 282017 | Accepted after revision: July 072018

This study is registered at clinicaltrials.gov with identifier number NCT01621321.

Author contributions: R. Agarwal conceived the study idea, was involved in patient management and drafted and revised the manuscript for intellectual content; S. Dhooria was involved in patient management and data collection, and revised the manuscript for intellectual content; I.S. Sehgal was involved in patient management and revised the manuscript; A.N. Aggarwal was involved in patient management, statistical analysis and revised the manuscript for intellectual content; M. Garg was involved in patient management and revised the manuscript; B. Saikia was involved in patient management and revised the manuscript; A. Chakrabarti was involved in patient management and revised the manuscript for intellectual content. 
Conflict of interest: R. Agarwal has received consultancy fees from Pulmatrix Inc., USA, during the conduct of the study.

Support statement: Cipla Pharmaceuticals (Mumbai, India) supported this work and provided the study drugs but had no role in the study design, data collection, data analysis or preparation of the manuscript. Funding information for this article has been deposited with the Crossref Funder Registry.

\section{References}

1 Agarwal R, Chakrabarti A, Shah A, et al. Allergic bronchopulmonary aspergillosis: review of literature and proposal of new diagnostic and classification criteria. Clin Exp Allergy 2013; 43: 850-873.

2 Agarwal R, Sehgal IS, Dhooria S, et al. Developments in the diagnosis and treatment of allergic bronchopulmonary aspergillosis. Expert Rev Respir Med 2016; 10: 1317-1334.

3 Oray M, Abu Samra K, Ebrahimiadib N, et al. Long-term side effects of glucocorticoids. Expert Opin Drug Saf 2016; 15: 457-465.

4 Agarwal R, Dhooria S, Singh Sehgal I, et al. A randomized trial of itraconazole vs prednisolone in acute-stage allergic bronchopulmonary aspergillosis complicating asthma. Chest 2018; 153: 656-664.

5 Agarwal R, Gupta D, Aggarwal AN, et al. Allergic bronchopulmonary aspergillosis: lessons from 126 patients attending a chest clinic in north India. Chest 2006; 130: 442-448.

6 Agarwal R, Aggarwal AN, Dhooria S, et al. A randomised trial of glucocorticoids in acute-stage allergic bronchopulmonary aspergillosis complicating asthma. Eur Respir J 2016; 47: 490-498.

7 Agarwal R, Dhooria S, Aggarwal AN, et al. Guidelines for diagnosis and management of bronchial asthma: joint ICS/NCCP (I) recommendations. Lung India 2015; 32: Suppl. 1, S3-S42.

8 Stevens DA, Schwartz HJ, Lee JY, et al. A randomized trial of itraconazole in allergic bronchopulmonary aspergillosis. N Engl J Med 2000; 342: 756-762.

9 Wark PA, Hensley MJ, Saltos N, et al. Anti-inflammatory effect of itraconazole in stable allergic bronchopulmonary aspergillosis: a randomized controlled trial. J Allergy Clin Immunol 2003; 111: 952-957.

10 Johnson LB, Kauffman CA. Voriconazole: a new triazole antifungal agent. Clin Infect Dis 2003; 36: 630-637.

Copyright (C)RS 2018 